\title{
Plasma Omentin-1 Levels Are Similar in Nondiabetic Metabolic Syndrome Patients and Healthy Subjects
}

\author{
Damla Coksert Kilic ${ }^{\mathrm{a}, \mathrm{b}}$, Aytekin Oguz ${ }^{\mathrm{a}}$, Mehmet Uzunlulua , Selda Celik ${ }^{\mathrm{a}}$, Gulsum Koroglu ${ }^{\mathrm{a}}$
}

\begin{abstract}
Background: Omentin-1 which is mainly produced by omental adipose tissue and associated with insulin dependent glucose uptake is less in obese patients than lean subjects. The aims of this study were; first, to compare plasma omentin-1 levels between nondiabetic metabolic syndrome patients and healthy controls and second, to investigate the association of plasma omentin-1 with metabolic parameters.
\end{abstract}

Methods: According to International Diabetes Federation (IDF) criteria, 50 nondiabetic metabolic syndrome patients (23 male, 27 female) and 30 healthy control subjects ( 9 male, 21 female) were recruited in this study. After 12 hour-fasting, plasma omentin-1 levels were measured by enzyme-linked immunosorbent assay (ELISA) in venous blood samples.

Results: There was no difference in plasma omentin-1 levels between metabolic syndrome patients and control subjects $(90.53$ $\pm 23.14 \mathrm{ng} / \mathrm{mL}, 87.83 \pm 17.10 \mathrm{ng} / \mathrm{mL}$ respectively; $\mathrm{p}=0.55) . \mathrm{In}$ all subjects, plasma omentin-1 was positively correlated with triglyceride and negatively correlated with HDL-C $(\mathrm{r}=0.24, \mathrm{p}=$ $0.029 ; \mathrm{r}=-0.62, \mathrm{p}=0.019$, respectively).

Conclusions: The results of this study showed that although the plasma omentin-1 levels were correlated with high triglyceride and low HDL-C levels which are two of five metabolic syndrome's parameters, the plasma omentin-1 levels are similar in metabolic

Manuscript accepted for publication Oct 5, 2011

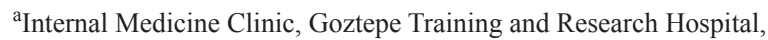
Istanbul, Turkey

bcorresponding author: Goztepe Training and Research Hospital, Internal Medicine Clinic, Baglarbasi mah. Feyzullah cad. Cam sk. Guven apt. No:20 D:1 Maltepe, Istanbul, Turkey.

Email: damlacoksert@yahoo.com

doi: $10.4021 /$ jem $46 \mathrm{w}$ syndrome patients and healthy controls. To investigate the tissue omentin-1 levels in the same groups may enlighten the topic.

Keywords: Omentin-1; Metabolic syndrome; Insulin resistance; Abdominal obesity; Obesity

\section{Introduction}

Metabolic syndrome (MetS) is a cluster of cardiometabolic risk factors characterized by abdominal obesity, insulin resistance and chronic inflammation[1]. Environmental and genetic factors are forming the base of the metabolic syndrome. Sedentary life style, physical inactivity, imbalanced and over feeding result in metabolic syndrome in subjects with genetically vulnerable to insulin resistance $[2,3]$. Even being clinically obese is not mandatory in the subjects with insulin resistance, there is generally abnormal fat distribution located at abdominal area. Many researchers claim that excessive visceral fat is more likely to be related with insulin resistance rather the other adipose tissue areas[4]. Visceral adipose tissue is especially more interesting than the subcutaneous adipose tissue, because recent researches showed that visceral fat is acting like an endocrine organ and it secretes varied adipokines which affect some steps in glucose and lipid metabolisms.

After the discovery of leptin and adiponectine, many other active substances secreted from visceral adipose tissue were detected [5-8]. Omentin (Intelectine)-1, which was firstly identified from the intestinal Paneth cells by Komiya, et al in 1998 and then from omental adipose tissue in 2004, is one of those adipokines $[9,10]$. Omentin could be lately detected in plasma. Omentin is a protein made of 313 monoacids. It is mostly produced in stromal cells of adipose tissue and less in intestines, lungs, heart, kidneys and muscle tissue. In vitro recombinant omentin therapy increases insulin dependent glucose uptake in subcutaneous and visceral adipose tissue. Omentin-1 levels of obese subjects were found to be less than those of lean subjects [11]. In addition, omentin gene is established in 1q22-q23 loci. These loci were found to be related with type 2 diabetes in varied populations. That 
Table 1. Demographic characteristics of the groups

\begin{tabular}{lllll}
\hline & Group 1 & Group 2 & Group 3 & p \\
\hline Age (years) & $45.96 \pm 4.85$ & $43.36 \pm 7.18$ & $43.27 \pm 5.36$ & 0.178 \\
Weight $(\mathrm{kg})$ & $85.34 \pm 14.9$ & $84.8 \pm 10.98$ & $69.39 \pm 14.5$ & $<0.0001$ \\
BMI $\left(\mathrm{kg} / \mathrm{m}^{2}\right)$ & $31.29 \pm 4.06$ & $30.27 \pm 4.08$ & $26.01 \pm 4.79$ & $<0.0001$ \\
Waist circumference (cm) & $105.08 \pm 11.51$ & $102.2 \pm 9$ & $84.83 \pm 13.72$ & $<0.0001$ \\
Hip cicumference (cm) & $113.6 \pm 7.07$ & $110.32 \pm 9.7$ & $104.4 \pm 10.97$ & 0.002 \\
Waist/Hip ratio & $0.92 \pm 0.07$ & $0.93 \pm 0.07$ & $0.81 \pm 0.07$ & $<0.0001$ \\
Systolic blood pressure (mmHg) & $132.4 \pm 16.90$ & $122.8 \pm 15.68$ & $116.67 \pm 13.98$ & 0.0027 \\
Diastolic blood pressure (mmHg) & $80 \pm 9.13$ & $76.2 \pm 10.54$ & $73.5 \pm 11.68$ & 0.085 \\
\hline
\end{tabular}

Group 1: Prediabetic metabolic syndrome patients; Group 2: Normoglycemic metabolic syndrome patients; Group 3: Control subjects

relationship gives rise to thought that Omentin-1 may play a role in the development of type 2 diabetes. In our study, we aimed to investigate the changes in plasma Omentin-1 levels in nondiabetic subjects with metabolic syndrome and healthy controls who were categorized according to their glucose levels and waist circumferences.

\section{Materials and Methods}

\section{Patients}

The study was conducted between April 2010 and May 2010 in Internal Medicine Outpatient Clinic of Goztepe Training and Research Hospital with 80 nondiabetic participants (50 metabolic syndrome patients and 30 healthy controls; 42 fe- male, 38 male; mean age $44.14 \pm 5.9$ years) who were more than 18 years old. Subjects with severe liver or kidney diseases, acute infection, type 1 or 2 diabetes, and subjects having diseases or using drugs that affect plasma glucose levels were excluded. Patients with metabolic syndrome were consecutively randomized according to their fasting plasma glucose (FPG) levels. All participants were categorized as Group 1 (Prediabetic patients with metabolic syndrome [FPG between 100 and $125 \mathrm{mg} / \mathrm{dL}, \mathrm{n}=25$ ]), Group 2 (Normoglycemic patients with metabolic syndrome $[\mathrm{FPG}<100$ $\mathrm{mg} / \mathrm{dL}, \mathrm{n}=25]$ ) and Group 3 (Healthy controls who were age and gender equivalents of the other groups with FPG < $100 \mathrm{mg} / \mathrm{dL}, \mathrm{n}=30$ ).

Local Ethics Committee approval was obtained for the research (Date: 06.04.2010, Decision No: 1/C). Written informed consents were taken from all participants according

Table 2. Basal biochemical parameters of the groups

\begin{tabular}{|c|c|c|c|c|}
\hline & Group 1 & Group 2 & Group 3 & $\mathbf{p}$ \\
\hline Fasting plasma glucose (mg/dl) & $108.92 \pm 7.2$ & $90.04 \pm 7.76$ & $91.67 \pm 9.6$ & $<0.0001$ \\
\hline Total cholesterol (mg/dl) & $217.72 \pm 52.42$ & $209.72 \pm 33.12$ & $205.77 \pm 36.48$ & 0.79 \\
\hline Triglyceride (mg/dl) & $213.64 \pm 114.32$ & $215.88 \pm 87.3$ & $94.07 \pm 38.26$ & $<0.0001$ \\
\hline HDL-C (mg/dl) & $47.04 \pm 10.46$ & $41.76 \pm 7.95$ & $61 \pm 14.18$ & $<0.0001$ \\
\hline LDL-C (mg/dl) & $150.04 \pm 41.7$ & $145.92 \pm 26.88$ & $134.07 \pm 29.37$ & 0.18 \\
\hline $\mathrm{HbA1C}(\%)$ & $5.85 \pm 0.43$ & $5.65 \pm 0.29$ & $5.44 \pm 0.31$ & 0.0001 \\
\hline
\end{tabular}

Group 1: Prediabetic metabolic syndrome patients; Group 2: Normoglycemic metabolic syndrome patients; Group 3: Control subjects 
Table 3. Plasma Omentin-1 levels of the groups

\begin{tabular}{llll}
\hline & Group 1 & Group 2 & Group 3 \\
\hline Plasma Omentin-1 $(\mathrm{ng} / \mathrm{mL})$ & $95.98 \pm 25.85$ & $85.08 \pm 19.05$ & $87.83 \pm 17.10$ \\
\hline
\end{tabular}

Group 1: Prediabetic metabolic syndrome patients; Group 2: Normoglycemic metabolic syndrome patients; Group 3: Control subjects

to 1964 Helsinki Declaration.

\section{Flow of the study}

Information on demographic characteristics and comorbid diseases of the patients were obtained. Blood pressure, height, weight, waist and hip circumferences of the patients were measured on physical examination by the same person. Body mass indexes (BMI) of the patients were calculated by using height and weight values. Clinical data and fasting biochemical parameters (fasting plasma glucose, total cholesterol, triglyceride, HDL-C, LDL-C, AST, ALT, creatinine, TSH and free T4) performed in the last week before the onset of the study were collected from the files of the subjects.

\section{Biochemical parameters}

Subjects accepted to participate in the study were invited to the clinic in the next morning after 12 hour fasting duration at 8:00 am. For measuring Omentin-1, venous blood samples were collected into plain tubes. Samples were centrifuged with $4000 \mathrm{cycle} / \mathrm{min}$ for 15 minutes and serum was separated and then stored at $-70^{\circ} \mathrm{C}$ until analysis for a week. Omentin-1 was studied on human Omentin 1 enzyme-linked immunosorbent assay (ELISA) kit with Pasteur ELISA device (APOTECH Corporation, Switzerland).

\section{Statistics}

All statistical analyses were made by using the software SPSS for Windows V15.0. Normality of distribution of variables was tested by Shapiro-Wilk and Kolmogorov-Smirnov tests. Subjects were compared for differences in anthropometric and biochemical data by two tailed Mann-Whitney U or Student's t test. Kruskal-Wallis test or Oneway ANOVA was performed for comparison of two or more independent samples. Correlations between variables were determined by Pearson correlation test or Spearman's Rho. Data are expressed as means \pm SD. A p value below 0.05 (two tailed) was considered to be statistically significant.

\section{Results}

Eighty subjects (42 female, 38 male) were categorized as Group 1 (Prediabetic patients with metabolic syndrome, $\mathrm{n}=$ 25), Group 2 (Normoglycemic patients with metabolic syndrome, $\mathrm{n}=25$ ) and Group 3 (Healthy controls who were age and gender equivalents of the other groups, $n=30$ ). There were no differences in age and gender of three groups $(\mathrm{p}=$ $0.257, p=0.101$, respectively). The demographic and biochemical parameters of the groups can be seen in Tables 1 and 2.

Omentin-1 levels were similar between patients with metabolic syndrome and control subjects $(90.53 \pm 23.14 \mathrm{ng} /$ $\mathrm{mL}, 87.83 \pm 17.10 \mathrm{ng} / \mathrm{mL}$, respectively; $\mathrm{p}=0.55)$. There was also no difference in plasma Omentin-1 level among three groups ( $\mathrm{p}=0.16$; Table 3 ).

Even the patients were categorized according to their BMI, plasma Omentin-1 levels were similar among three groups ( $p=0.80$; Table 4$)$. There was no significant difference in plasma Omentin-1 levels of patients with FPG $<100$ $\mathrm{mg} / \mathrm{dL}$ and $\geq 100 \mathrm{mg} / \mathrm{dL}$ ( $\mathrm{p}=0.073$; Fig. 1$)$.

Plasma Omentin-1 level was positively correlated with triglyceride and negatively correlated with HDL-C $(r=0.24$, $\mathrm{p}=0.029 ; \mathrm{r}=-0.62, \mathrm{p}=0.019$, respectively; Fig. 2). In female subjects, plasma Omentin-1 was positively correlated with systolic blood pressure, FPG and HbA1c $(r=0.29, \mathrm{p}=$ $0.046 ; \mathrm{r}=0.33, \mathrm{p}=0.023 ; \mathrm{r}=0.31, \mathrm{p}=0.032$, respectively). However, there was only a negative correlation between plasma Omentin-1 and HDL-C in male subjects $(\mathrm{r}=0.39$, $\mathrm{p}=0.027)$.

Table 4. Plasma Omentin-1 levels according to BMI groups

\begin{tabular}{lllr}
\hline & BMI groups & & \\
\hline & $<25 \mathrm{~kg} / \mathrm{m}^{2}$ & $25-29.9 \mathrm{~kg} / \mathrm{m}^{2}$ & $\geq 30 \mathrm{~kg} / \mathrm{m}^{2}$ \\
Plasma Omentin-1 $(\mathrm{ng} / \mathrm{mL})$ & $86.18 \pm 14.67$ & $90.60 \pm 23.62$ & $89.82 \pm 20.82$ \\
\hline
\end{tabular}




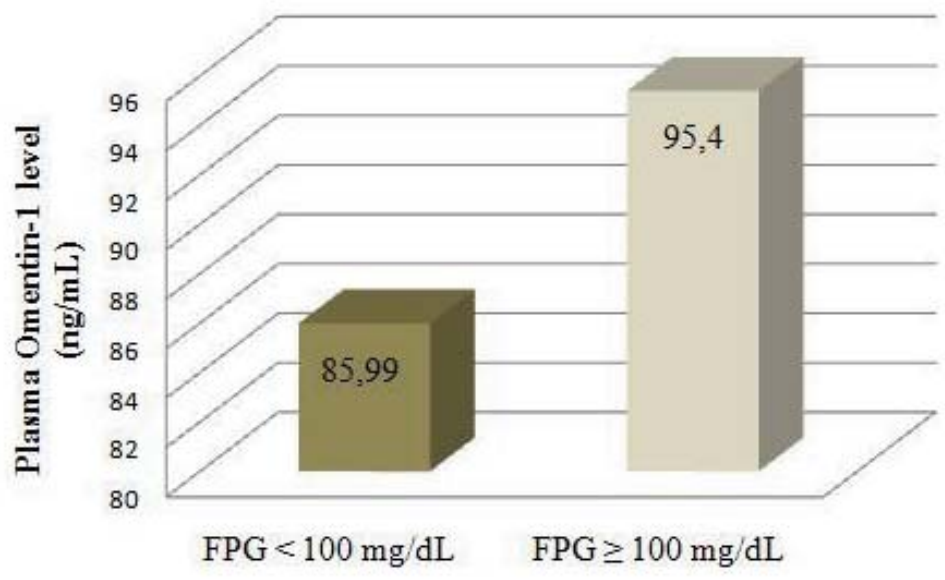

Figure 1. Plasma Omentin-1 levels according to fasting plasma glucose categories
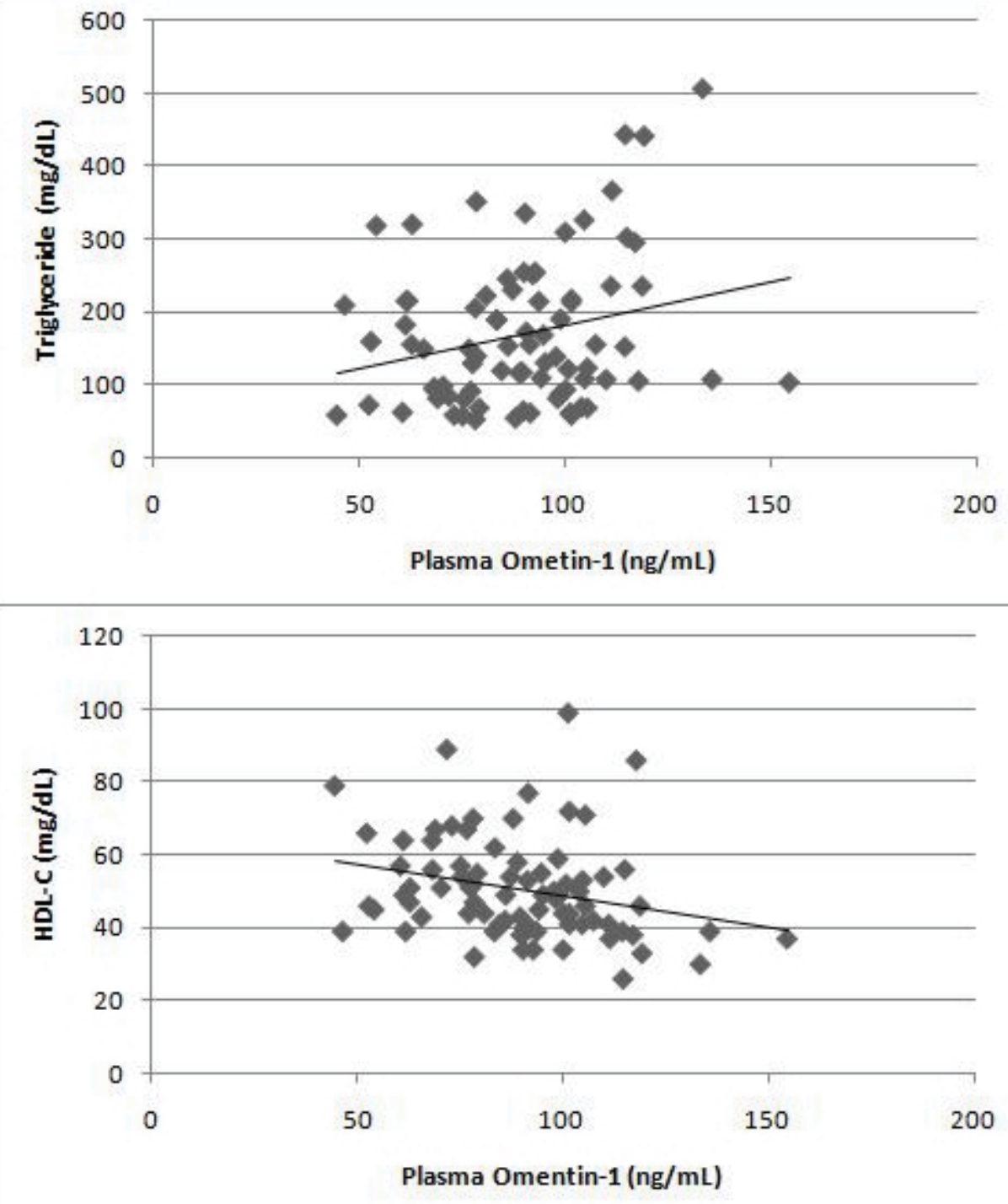

Figure 2. The correlation of plasma Omentin-1 with triglyceride and HDL-C in all patients 


\section{Discussion}

Abdominal obesity is one of the dominant characteristics of metabolic syndrome. According to the results of the recent study, even there was a correlation between plasma Omentin-1 and triglyceride and HDL-C, plasma Omentin-1 levels were similar in patients with metabolic syndrome and healthy subjects regardless of their BMI.

Omentin is an adipokine that regulates insulin activity [12]. It increases insulin stimulated uptake of glucose and Akt phosphorylation in adipocytes. We found no difference between subjects with metabolic syndrome and healthy controls. On the other hand, visceral Omentin-1 and plasma Omentin levels in obese subjects were less than the lean subjects in the study of Souza Batista, et al [13]. However, unlike our study, subjects were not evaluated according to the presence of metabolic syndrome in this study, so they ignored some of the parameters of the metabolic syndrome and they also found that plasma Omentin-1 was positively correlated with HDL-C. They have used a part of the participants of Amish Family Diabetes Study [14] who were relatives to each other. Different than that study, our sample was randomly selected from whole population. One of the differences between studies was the technique used to determine Omentin-1 levels. De Souza Batista, et al used western blot technique, but we studied Omentin-1 with ELISA kit. Even the western blot technique can determine tiny levels of protein, it is obviously more difficult and less sensitive than ELISA technique to perform and also open to do errors.

Plasma Omentin-1 levels were similar between genders in our study. In the study of de Souza Batista, et al, plasma Omentin-1 was higher in women, on the other hand MorenoNavarrete, et al [15] who used ELISA technique, found that plasma levels of Omentin-1 is higher in men. It shows us that plasma Omentin levels can be affected from other factors rather than gender and we can see differences among studies. On the other hand, the design of the study of MorenoNavarrete, et al is different than ours. It is a weight loss study with less subjects $(n=35)$ and they only took obese people. Even they found negative correlation between plasma Omentin-1 and weight, BMI and waist circumference, there wasn't a correlation neither in obese $(n=32)$ nor in abdominal obese subjects $(n=63)$ in our study.

In another study, plasma Omentin-1 levels were evaluated in women with polycystic ovary syndrome $(n=20)$ which includes obesity and insulin resistance as important components [16]. Plasma Omentin-1 levels were less in women with polycystic ovary syndrome and there was a negative correlation between plasma Omentin-1 and BMI. According to our findings, there was no such a correlation even in female subjects $(\mathrm{n}=42)$.

In a recent study of Pan, et al, they investigated plasma Omentin-1 levels (with ELISA technique) in subjects with normal glucose tolerance, impaired glucose regulation and treatment-naive newly diagnosed type 2 diabetes [17]. In subjects with either impaired glucose regulation or type 2 diabetes, plasma omentin-1 levels were less than the control group. Differently, plasma Omentin-1 levels were similar in prediabetic subjects and control group in our findings. However, we did not perform oral glucose tolerance test (OGTT) to determine glucose regulation and we evaluated the situation only according to FPG, so there are 13 normoglycemic, healthy subjects who do not carry all metabolic syndrome criteria although they have high waist circumference and 25 normoglycemic metabolic syndrome patients in our study. We should keep in mind that the results might be different if we performed OGTT.

It is the first study which investigated the relationship of metabolic syndrome parameters with plasma Omentin-1 in metabolic syndrome patients and healthy subjects aged between 18-65 years old. The limitations were that we did not perform OGTT or measure insulin levels. On the other hand, to take nondiabetic metabolic syndrome patients and to exclude subjects who were taking drugs affecting glucose metabolism were the strong sides of the study.

As a result, plasma Omentin-1 levels are similar among prediabetic and normoglycemic metabolic syndrome subjects and healthy controls. Metabolic syndrome includes many cardiovascular risk factors other than the abdominal obesity, so many conflicting variables can affect the results. It puts forth the necessity of future studies for consideration to enlighten the association of metabolic syndrome with Omentin-1.

\section{Conflict of Interest}

The authors declare no conflict of interest.

\section{References}

1. Grundy SM, Cleeman JI, Daniels SR, Donato KA, Eckel RH, Franklin BA, Gordon DJ, et al. Diagnosis and management of the metabolic syndrome: an American Heart Association/National Heart, Lung, and Blood Institute Scientific Statement. Circulation. 2005;112(17):27352752.

2. Reaven GM. Banting lecture 1988. Role of insulin resistance in human disease. Diabetes. 1988;37(12):15951607.

3. Meigs JB. Epidemiology of the metabolic syndrome, 2002. Am J Manag Care. 2002;8(11 Suppl):S283-292; quiz S293-286.

4. Brochu M, Starling RD, Tchernof A, Matthews DE, Garcia-Rubi E, Poehlman ET. Visceral adipose tissue is an independent correlate of glucose disposal in older obese 
postmenopausal women. J Clin Endocrinol Metab. 2000;85(7):2378-2384.

5. Hampton T. Scientists study fat as endocrine organ. JAMA. 2006;296(13):1573-1575.

6. Myers MG, Cowley MA, Munzberg H. Mechanisms of leptin action and leptin resistance. Annu Rev Physiol. 2008;70:537-556.

7. Leinninger GM, Myers MG, Jr. LRb signals act within a distributed network of leptin-responsive neurones to mediate leptin action. Acta Physiol (Oxf). 2008;192(1):4959.

8. Lu JY, Huang KC, Chang LC, Huang YS, Chi YC, Su TC, Chen CL, et al. Adiponectin: a biomarker of obesity-induced insulin resistance in adipose tissue and beyond. J Biomed Sci. 2008;15(5):565-576.

9. Komiya T, Tanigawa Y, Hirohashi S. Cloning of the novel gene intelectin, which is expressed in intestinal paneth cells in mice. Biochem Biophys Res Commun. 1998;251(3):759-762.

10. Scherer PE, Williams S, Fogliano M, Baldini G, Lodish HF. A novel serum protein similar to $\mathrm{C} 1 \mathrm{q}$, produced exclusively in adipocytes. J Biol Chem. 1995;270(45):2674626749.

11. Schaffler A, Neumeier M, Herfarth H, Furst A, Scholmerich J, Buchler C. Genomic structure of human omentin, a new adipocytokine expressed in omental adipose tissue. Biochim Biophys Acta. 2005;1732(1-3):96-102.

12. Yang RZ, Lee MJ, Hu H, Pray J, Wu HB, Hansen BC,
Shuldiner AR, et al. Identification of omentin as a novel depot-specific adipokine in human adipose tissue: possible role in modulating insulin action. Am J Physiol Endocrinol Metab. 2006;290(6):E1253-1261.

13. de Souza Batista CM, Yang RZ, Lee MJ, Glynn NM, Yu DZ, Pray J, Ndubuizu K, et al. Omentin plasma levels and gene expression are decreased in obesity. Diabetes. 2007;56(6):1655-1661.

14. Hsueh WC, Mitchell BD, Aburomia R, Pollin T, Sakul H, Gelder Ehm M, Michelsen BK, et al. Diabetes in the Old Order Amish: characterization and heritability analysis of the Amish Family Diabetes Study. Diabetes Care. 2000;23(5):595-601.

15. Moreno-Navarrete JM, Catalan V, Ortega F, GomezAmbrosi J, Ricart W, Fruhbeck G, Fernandez-Real JM. Circulating omentin concentration increases after weight loss. Nutr Metab (Lond). 2010;7:27.

16. Tan BK, Adya R, Farhatullah S, Lewandowski KC, O'Hare P, Lehnert H, Randeva HS. Omentin-1, a novel adipokine, is decreased in overweight insulin-resistant women with polycystic ovary syndrome: ex vivo and in vivo regulation of omentin-1 by insulin and glucose. Diabetes. 2008;57(4):801-808.

17. Pan HY, Guo L, Li Q. Changes of serum omentin-1 levels in normal subjects and in patients with impaired glucose regulation and with newly diagnosed and untreated type 2 diabetes. Diabetes Res Clin Pract. 2010;88(1):2933. 